\title{
Síndrome similar a hiperostosis esquelética difusa idiopática toracolumbar asociada a isotretinoína: reporte de un caso en una paciente joven
}

\author{
Jessica E. Figueroa-Estrada ${ }^{1 *}$, Citlallyc Gómez-Ruiz ${ }^{2}$, Julio Palacios-Juárez ${ }^{3}$, Jesús Morales-Maza ${ }^{4}$ \\ y Ludivina A. Cortés-Martínez ${ }^{5}$ \\ ${ }^{1}$ Clínica para el Diagnóstico y Tratamiento de las Enfermedades Reumáticas, Ciudad de México, Méx.; ${ }^{2}$ Departamento de Reumatología, Hospital General de México, \\ Ciudad de México, Méx., ${ }^{3}$ Departamento de Cirugía Reconstructiva, Hospital Regional de Alta Especialidad de Ixtapaluca, Ixtapaluca, Méx.; ${ }^{4}$ Cirugía, Instituto Nacional \\ de Ciencias Médicas y Nutrición Salvador Zubirán, Ciudad de México, Méx.; ${ }^{5}$ Departamento de Anestesiología, Hospital Ángeles Lomas, Ciudad de México, Méx.
}

\begin{abstract}
We report the case of a 28-year-old woman with pain in the dorsal and lumbar spine of 2 years of evolution with a history of acne treatment with isotretinoin for 6 years. The findings by computed tomography showed skeletal vertebral hyperostosis. The use of vitamin A and its derivatives, such as isotretinoin, has been associated to the development of skeletal hyperostosis, and its severity seems to be related to the duration of treatment. Currently, the use of this drug is widespread in young patients with acne, even without medical prescription, unaware of the effects it can cause at the osteoarticular level. (Hosp Med Clin Manag. 2019;12:120-3)

Corresponding author: Jessica E. Figueroa-Estrada, jess.fig06@gmail.com
\end{abstract}

Key words: Skeletal hyperostosis. Isotretinoin. Spine.

\section{RESUMEN}

Presentamos el caso de una mujer de 28 años con dolor en la columna dorsal y lumbar de dos años de evolución con antecedente de tratamiento de acné a base de isotretinoína durante seis años. Los hallazgos por tomografía computarizada demostraron hiperostosis esquelética vertebral. El uso de vitamina A y sus derivados como la isotretinoína se ha asociado al desarrollo de hiperostosis esquelética y su gravedad parece estar relacionada con la duración del tratamiento. Actualmente el uso de dicho fármaco se ha generalizado en pacientes jóvenes con acné, incluso sin prescripción médica, desconociendo los efectos que puede causar a nivel osteoarticular.

Palabras clave: Hiperostosis esquelética. Isotretinoína. Columna vertebral.

\section{CASO CLÍNICO}

Se trata de una mujer de 28 años de edad con cuadro clínico de dos años de evolución con dolor de aumento progresivo en columna torácica y lumbar de intensidad 8/10 constante, opresivo, sin irradiaciones; no presentaba exacerbantes y mejoraba parcialmente con antiinflamatorios no esteroideos y opioides por vía oral. No

\section{Correspondencia:}

*Jessica E. Figueroa-Estrada

E-mail: jess.fig06@gmail.com
Date of reception: $05-07-2018$

Date of acceptance: 12-08-2019

DOI: 10.24875/HMCM.19000139 
presentaba rigidez matutina ni cambios asociados con actividad o reposo. Negaba dolor en columna cervical o en articulaciones sacroilíacas. Sin antecedente familiar de síndrome metabólico. Como único antecedente de importancia, se había automedicado con isotretinoína durante seis años con dosis de $20 \mathrm{mg}$ al día por vía oral $(0.25 \mathrm{mg} / \mathrm{kg})$ por presentar acné papulopustuloso. La paciente niega el consumo de algunos otros medicamentos o multivitamínicos. A la exploración física presentaba talla de $1.70 \mathrm{~m}$ y pesaba $77 \mathrm{~kg}$, con índice de masa corporal de 26.64. A la presión digital en la columna dorsal presentaba dolor de predominio en vértebras T2 a T5 y a nivel lumbar presentaba leve dolor en L1 y L2. Sin evidencia de limitación en los movimientos de la columna.

Se realizaron exámenes de laboratorio que reportaban velocidad de sedimentación globular y proteína $\mathrm{C}$ reactiva en parámetros normales; además, la paciente era HLA-B27 negativo y la citometría hemática y la química sanguínea se encontraban dentro de rangos normales. La radiografía de cadera no mostraba datos de sacroilitis. Se realizó tomografía computarizada de columna con evidencia de hiperostosis de vértebras torácicas T2-T6 y lumbares L1-L2 (Fig. 1).

Se realizó el diagnóstico de síndrome similar a hiperostosis esquelética difusa idiopática (HEDI). De acuerdo con el algoritmo de Naranjo sobre reacción adversa a medicamentos se obtuvo una puntuación total de 5 puntos (diagnóstico probable) ${ }^{1}$. Se inició tratamiento a base de antiinflamatorios no esteroideos por vía oral para lograr control del dolor de acuerdo con la escala de la Organización Mundial de la Salud. Se indicó suspender la isotretinoína y se inició tratamiento fisioterapéutico. A seis meses de iniciado el tratamiento se le ha dado seguimiento al caso en consultas periódicas, con mejoría parcial del dolor, actualmente con intensidad 2/10 de manera intermitente.

\section{DISCUSIÓN}

La HEDI fue descrita en 1955 por Forestier y RotésQuerol2. A pesar de haber pasado casi 60 años desde su descripción original continúa siendo una entidad poco conocida. Se caracteriza por calcificación progresiva y osificación de ligamentos con evidencia de entesis. La prevalencia promedio reportada es del 10\% en la población mayor de 50 años de edad, siendo

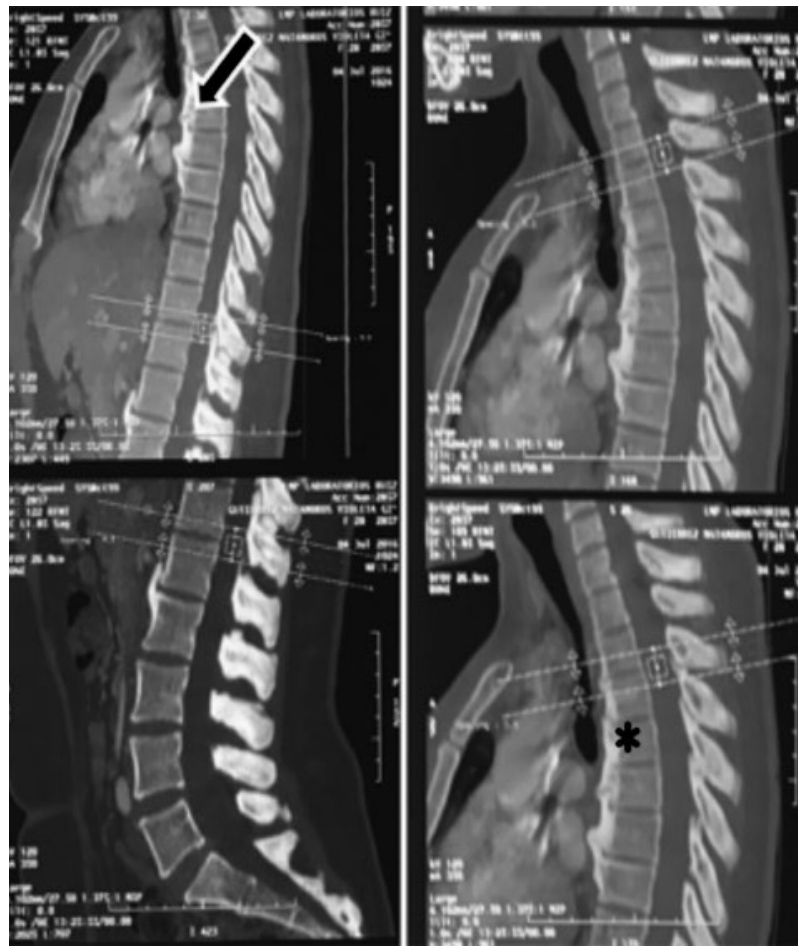

Figura 1. Tomografía de columna toracolumbar. Flecha negra y asterisco: Se observa calcificación y osificación a lo largo de la región ventral los cuerpos vertebrales de T2 a T6 y L1 a L2 con preservación de su altura.

muy rara su presentación en menores de 40 años $^{3}$. Es una afección asintomática en muchos individuos y puede ser difícil distinguirla de los cambios osteodegenerativos en los ancianos, en quienes es mucho más común.

Se ha tratado de establecer la fisiopatología de la HEDI y se ha establecido que los niveles séricos del inhibidor de osteogénesis natural Dickkopf-1 se encuentran disminuidos. Sin embargo, a pesar de que esto podría contribuir a la osificación vertebral, pero este hallazgo bioquímico no se encuentra de manera constante en todos los pacientes estudiados ${ }^{4}$.

Los criterios diagnósticos de HEDI descritos por Resnick y Niwayama ${ }^{5}$ son calcificación y osificación del ligamento anterolateral de por lo menos cuatro cuerpos vertebrales contiguos, preservación de la altura del disco intervertebral en las áreas involucradas, ausencia de cambios radiográficos de enfermedad degenerativa y ausencia de anquilosis ósea y afección de sacroilíacas. Otro autor, en un intento por detectar de manera más temprana esta enfermedad, disminuyó el umbral de la afectación vertebral a solo tres vértebras afectadas ${ }^{6}$. 
Tabla 1. Algoritmo de Naranjo para evaluar la causalidad de una reacción adversa a medicamento

\begin{tabular}{|c|c|c|c|c|}
\hline $\begin{array}{l}\text { Algoritmo de Naranjo para evaluar la causalidad de } \\
\text { una reacción adversa a un fármaco }\end{array}$ & Sí & No & No se sabe & $\begin{array}{l}\text { Puntuación en nuestro } \\
\text { paciente }\end{array}$ \\
\hline $\begin{array}{l}\text { 1. ¿Existen informes previos concluyentes acerca de } \\
\text { esta reacción? }\end{array}$ & +1 & 0 & 0 & +1 \\
\hline $\begin{array}{l}\text { 2. El acontecimiento adverso ¿apareció después de que } \\
\text { se administrara el fármaco sospechoso? }\end{array}$ & +2 & -1 & 0 & +2 \\
\hline $\begin{array}{l}\text { 3. La reacción adversa ¿mejoró al interrumpirse el } \\
\text { tratamiento o cuando se administró un antagonista } \\
\text { específico? }\end{array}$ & +1 & 0 & 0 & +1 \\
\hline $\begin{array}{l}\text { 4. ¿Reapareció la reacción adversa cuando se volvió a } \\
\text { administrar el fármaco? }\end{array}$ & +2 & -1 & 0 & 0 \\
\hline $\begin{array}{l}\text { 5. ¿Hay otras causas (distintas de la administración del } \\
\text { fármaco) que puedan por sí mismas haber ocasionado } \\
\text { la reacción? }\end{array}$ & -1 & +2 & 0 & -1 \\
\hline $\begin{array}{l}\text { 6. ¿Reapareció la reacción cuando se administró un } \\
\text { placebo? }\end{array}$ & -1 & +1 & 0 & 0 \\
\hline $\begin{array}{l}\text { 7. ¿Se ha detectado el fármaco en sangre (o en otros } \\
\text { humores) en una concentración cuya toxicidad es } \\
\text { conocida? }\end{array}$ & +1 & 0 & 0 & +1 \\
\hline $\begin{array}{l}\text { 8. ¿Aumentó la gravedad de la reacción al aumentarse } \\
\text { la dosis o disminuyó al reducirla? }\end{array}$ & +1 & 0 & 0 & 0 \\
\hline $\begin{array}{l}\text { 9. ¿Había sufrido el paciente una reacción similar al } \\
\text { mismo fármaco o a fármacos análogos en alguna } \\
\text { exposición previa? }\end{array}$ & +1 & 0 & 0 & 0 \\
\hline $\begin{array}{l}\text { 10. ¿Se confirmó el acontecimiento adverso mediante } \\
\text { pruebas objetivas? }\end{array}$ & +1 & 0 & 0 & +1 \\
\hline Puntuación total & & & & 5 puntos \\
\hline
\end{tabular}

Las categorías correspondientes a la puntuación total son las siguientes. La reacción adversa al medicamento es: segura (> 9), probable (5-8), posible (1-4) 0 improbable (0).

Por otro lado, desde hace muchos años se ha asociado el consumo de retinoides con entesopatía ${ }^{7-10}$. A esta afección musculoesquelética se la ha denominado síndrome similar a HEDI (DISH-like) que ocurre en pacientes que han recibido dosis altas de vitamina A por vía oral y sus compuestos, incluyendo etretinato e isotretinoína. Nosotros realizamos una evaluación de causalidad utilizando el algoritmo de Naranjo, obteniendo una puntuación de 5 (reacción adversa al medicamento probable). El algoritmo de Naranjo emplea 10 preguntas y da una aproximación de causalidad entre la exposición a un fármaco y algún efecto adverso ${ }^{1}$. Agregamos dicho algoritmo en la tabla 1.

Este síndrome fue descrito por primera vez por Pittsley y Yoder en 1983, quienes describieron el desarrollo de un trastorno de osificación similar a HEDI en cuatro pacientes que tomaban isotretinoína como tratamiento de la ictiosis ${ }^{11}$. Otros estudios han reportado una alta incidencia de efectos esqueléticos en la terapia con isotretinoína, dando como resultado una progresión radiográfica con hiperostosis cada vez más extensa con la duración del tratamiento relacionado con dosis altas o con la dosis acumulada de 150,060 mg durante 2.9 años ${ }^{12,13}$, involucrando los cuerpos vertebrales y los ligamentos longitudinales anterior y posterior, y progresando hacia el desarrollo de hiperostosis apendicular que aparece después de tres a cinco años de tratamiento ${ }^{14-16}$. Por otra parte, algunos estudios en donde se han analizado los efectos adversos en relación con las dosis empleadas a corto plazo de isotretinoína para el acné confirman que un curso de cuatro meses a dosis de $0.5 \mathrm{mg} / \mathrm{kg}$ diarios no causa anomalías radiológicas significativas a largo plazo ${ }^{17}$. Aún no está claro si existe un efecto hiperostósico acumulativo de los ciclos cortos repetidos con dosis bajas de isotretinoína.

La teoría de la hiperostosis asociada al tratamiento con retinoides está fundamentada debido a que el ácido retinoico es esencial para la diferenciación de las células mesenquimatosas y el desarrollo embrionario del 
esqueleto, así como en el metabolismo óseo normal ${ }^{18}$. Se ha reportado engrosamiento cortical o reacciones periósticas de los huesos largos en niños después de la ingestión a largo plazo de grandes cantidades de suplementos con vitamina $A$ (retinol) ${ }^{17}$. La isotretinoína es un retinoide sintético utilizado para el tratamiento de acné y es posible que su uso pueda causar proliferación y diferenciación de las células madre mesenquimales en osteoblastos en las entesis, lo que conduce a la osificación de estas ${ }^{18}$.

\section{CONCLUSIÓN}

Es importante que dermatólogos, médicos generales y reumatólogos reconozcan los cambios hiperostósicos inducidos por este fármaco como evento adverso con características similares a la hiperostosis esquelética idiopática difusa, ya que actualmente se utiliza de forma irracional en pacientes de cualquier edad con acné, por lo que podemos esperar un incremento importante en la incidencia de esta enfermedad, sobre todo en pacientes jóvenes.

En el caso que hemos reportado, los autores consideramos que la exposición durante largo tiempo a altas dosis de isotretinoína se encuentra relacionada a la hiperostosis avanzada de esta paciente joven.

\section{AUTORÍA}

Jessica E. Figueroa-Estrada y Citlallyc Gómez-Ruiz realizaron el diagnóstico, tratamiento y han dado el seguimiento de la paciente, así como la revisión y el diseño del manuscrito. Julio Palacios-Juárez, Jesús MoralesMaza y Ludivina A. Cortés-Martínez realizaron revisión de la literatura y diseñaron el manuscrito. Todos los autores revisaron y aprobaron la versión final del manuscrito.

\section{CONFLICTO DE INTERESES}

Los autores declaran no tener conflicto de intereses.

\section{RESPONSABILIDADES ÉTICAS}

\section{Confidencialidad de los datos}

Los autores declaran que han seguido los protocolos de su centro de trabajo sobre la publicación de datos de pacientes.

\section{Derecho a la privacidad y consentimiento informado}

Los autores han obtenido el consentimiento informado de los pacientes y/o sujetos referidos en el artículo.

\section{BIBLIOGRAFÍA}

1. Naranjo CA, Busto U, Sellers EM, Sandor P, Ruiz I, Roberts EA, et al. A method for estimating the probability of adverse drug reactions. Clin Pharmacol Ther. 1981;30:239-45.

2. Forestier J, Rotes-Querol J. Senile ankylosing hyperostosis of the spine. Ann Rheum Dis. 1950;9(4):321-30.

3. Mader R, Verlaan JJ, Eshed I, Jacome BA, Puttini PS, Atzeni F, et al. Diffuse idiopathic skeletal hyperostosis (DISH): Where we are now and where to go next. RMD Open. 2017;3(1):e000472.

4. Mazières B. Diffuse idiopathic skeletal hyperostosis (Forestier-Rotes Quero disease): What's new? Joint Bone Spine. 2013;80(5):466-70.

5. Resnick D, Niwayama G. Radiographic and pathologic features of spina involvement in diffuse idiopathic skeletal hyperostosis (DISH). Radiology. 1976;119(3):559-68

6. Utsinger PD. Diffuse idiopathic skeletal hyperostosis. Clin Rheum Dis. 1985; 11:325-51.

7. Stitik TP, Nadler SF, Foye PM, Juvan L. Greater trochanter enthesopathy: an example of "short course retinoid enthesopathy": a case report. Am J Phys Med Rehabil. 1999;78(6):571-6.

8. Brandt JR, Mick TJ. Extraspinal enthesopathy caused by isotretinoin therapy. J Manipulative Physiol Ther. 1999:22(6):417-20.

9. White SI, MacKie RM. Bone changes associated with oral retinoid therapy. Pharmacol Ther. 1989;40(1):137-44.

10. Melnik B, Plewig G. Unwanted bone changes in systemic treatment with synthetic retinoids. Hautarzt. 1987;38(4):193-7.

11. Pittsley RA, Yoder FW. Retinoid hyperostosis. Skeletal toxicity associated with long-term administration of 13-cis-retinoic acid for refractory ichthyosis. N Engl J Med. 1983:308(17):1012-4.

12. Gerber LH, Helfgott RK, Gross EG, Hicks JE, Ellenberg SS, Peck GL. Vertebral abnormalities associated with synthetic retinoid use. J Am Acad Dermatol. 1984;10(5 Pt 1):817-23.

13. Ellis CN, Pennes DR, Martel W, Voorhees JJ. Radiographic bone surveys after isotretinoin therapy for cystic acne. Acta Derm Venereol. 1985;65(1):83-5

14. Ellis CN, Pennes DR, Hermann RC, Blauvelt A, Martel W, Voorhees JJ. Long-term radiographic follow-up after isotretinoin therapy. J Am Acad Dermatol. 1988;18(6):1252-61.

15. Pennes DR, Martel W, Ellis CN, Voorhees JJ. Evolution of skeletal hyperostoses caused by 13-cis-retinoic acid therapy. AJR Am J Roentgenol. 1988;151(5):967-73.

16. DiGiovanna JJ. Isotretinoin effects on bone. J Am Acad Dermatol. 2001;45(5):S176-82.

17. Kilcoyne RF, Cope R, Cunningham W, Nardella FA, Denman S, Franz TJ, et al. Minimal spinal hyperostosis with low-dose isotretinoin therapy. Invest Radiol. 1986;21(1):41-4.

18. Troillet N, Gerster JC. Forestier disease and metabolism disorders. A prospective controlled study of 25 cases. Rev Rhum Ed Fr. 1993;60(4):274-9. 